PROCEEDINGS OF THE

AMERICAN MATHEMATICAL SOCIETY

Volume 134, Number 4, Pages 1039-1044

S 0002-9939(05)08301-2

Article electronically published on November 17, 2005

\title{
THE LIPSCHITZ FREE BANACH SPACES OF $C(K)$-SPACES
}

\author{
YVES DUTRIEUX AND VALENTIN FERENCZI
}

(Communicated by David Preiss)

\begin{abstract}
The aim of this note is to prove that if $K$ is any infinite metric compact space, then the Lipschitz free spaces of $C(K)$ and $c_{0}$ are isomorphic. This gives an example of non-Lipschitz-homeomorphic Banach spaces whose free Lipschitz spaces are isomorphic. We also derive some results about Lipschitz homogeneity for Banach spaces, from the results of G. Godefroy and N. J. Kalton on Lipschitz free Banach spaces.
\end{abstract}

\section{The LipsChitZ FReE BANACH SPACE $\mathcal{F}_{c_{0}}$}

We refer to [5] for the background of this note. Let $X$ be a metric space with a special point 0 . We denote by $\operatorname{Lip}_{0}(X)$ the space of Lipschitz functions from $X$ to $\mathbb{R}$ which vanish at 0 . The norm of a function $f \in \operatorname{Lip}_{0}(X)$ is the Lipschitz constant

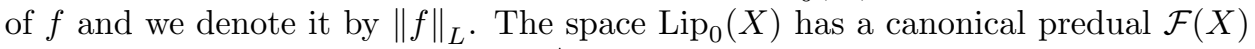
which is the linear span in $\operatorname{Lip}_{0}(X)^{*}$ of the evaluation functionals $\delta_{x}: f \mapsto f(x)$ for $x \in X$. The space $\mathcal{F}(X)$ is called the Lipschitz free space of $X$. It is clearly invariant under Lipschitz homeomorphisms. So it is natural to ask how much the Lipschitz free space of a Banach space $X$ captures of the Lipschitz structure of $X$. To the best of the authors' knowledge, it was not even known if two Banach spaces could have the same Lipschitz free space without being Lipschitz homeomorphic. In this note, we prove that, for any infinite compact metric space $K$, the spaces $\mathcal{F}(C(K))$ and $\mathcal{F}\left(c_{0}\right)$ are isomorphic. In fact we prove that the Banach-Mazur distance between $\mathcal{F}(C(K))$ and $\mathcal{F}\left(c_{0}\right)$ is uniformly bounded. By Theorem 3.1 in [7, we know that $C(K)$ and $c_{0}$ are not uniformly homeomorphic (and thus not Lipschitz homeomorphic) if $C(K)$ is not linearly isomorphic to $c_{0}$. Hence this gives an example of a couple of Banach spaces which have isomorphic Lipschitz free spaces but are not uniformly homeomorphic.

Let us begin with a definition. Let $X_{1}, X_{2}, \ldots$ be Banach spaces and $X=$ $\left(\oplus X_{n}\right)_{c_{0}}$. We denote by $\hat{X}_{n}$ the subspace of $X$ equal to $\sum_{i=1}^{n-1}\{0\} \oplus X_{n} \oplus \sum_{i=n+1}^{\infty}\{0\}$. The set $\bigcup \hat{X}_{n}$ is denoted by $X_{n}$ and called the cross of the $X_{n}$ 's. It is equipped with the metric $d$ inherited by the norm of $X$.

Proposition 1. Let $X_{1}, X_{2}, \ldots$ be Banach spaces. The Banach spaces $\left(\bigoplus \mathcal{F}\left(X_{n}\right)\right)_{\ell_{1}}$ and $\mathcal{F}\left(X_{n}\right)$ are isomorphic.

Received by the editors October 5, 2004

2000 Mathematics Subject Classification. Primary 46B03.

Part of this article was written when the second author was at the University of São Paulo, under the FAPESP grant 2002/09662-1. 
Proof. The dual spaces of $A=\left(\oplus \mathcal{F}\left(X_{n}\right)\right)_{\ell_{1}}$ and $B=\mathcal{F}\left(X_{n}\right)$ are respectively $A^{*}=\left(\bigoplus \operatorname{Lip}_{0}\left(X_{n}\right)\right)_{\ell_{\infty}}$ and $B^{*}=\operatorname{Lip}_{0}\left(X_{n}\right)$.

We define the map $\Phi: A^{*} \rightarrow B^{*}$ by $\Phi(f)=g$ where $f=\left(f_{n}\right)_{n \in \mathbb{N}}$ and $g(x)=$ $f_{n}(x)$ if $x \in \hat{X}_{n}$ (here, we identify $X_{n}$ and $\hat{X}_{n}$, which does not carry any confusion).

Let $x \in \hat{X}_{n}$ and $y \in \hat{X}_{m}$ be two points of the cross. If $n=m$, we have

$$
|\Phi(f)(x)-\Phi(f)(y)| \leq\left\|f_{n}\right\|_{L} \cdot\|x-y\|_{X_{n}} \leq\|f\|_{\infty} d(x, y) .
$$

On the other hand, when $n \neq m$, we have

$$
|\Phi(f)(x)-\Phi(f)(y)| \leq\left\|f_{n}\right\|_{L} \cdot\|x\|_{X_{n}}+\left\|f_{m}\right\|_{L} \cdot\|y\|_{X_{m}} \leq 2\|f\|_{\infty} d(x, y) .
$$

Hence, $\|\Phi(f)\|_{L} \leq 2\|f\|_{\infty}$ for all $f \in A^{*}$, and thus $\Phi$ has norm at most 2. The inverse of $\Phi$ is given by $\Phi^{-1}(f)=\left(\left.f\right|_{X_{n}}\right)_{n \in \mathbb{N}}$ and it has clearly norm at most 1 . Moreover $\Phi$ is weak* to weak* continuous so it induces an isomorphism between $A$ and $B$.

The next step in our proof will be to show that $\left(\bigoplus \mathcal{F}\left(c_{0}\right)\right)_{\ell_{1}}$ is isomorphic to $\mathcal{F}\left(c_{0}\right)$. In order to have that, we need a lemma:

Lemma 2. Let $X_{1}, X_{2}, \ldots$ be Banach spaces and $X=\left(\bigoplus X_{n}\right)_{c_{0}}$. Then there is a 3-Lipschitz retraction from $X$ onto $X_{n}$ which maps 0 to 0 .

Proof. For any integer $q$, let $p_{q}$ denote the canonical projection from $X$ onto $\left(\bigoplus_{n \neq q} X_{n}\right)_{c_{0}}$, that is to say, for any vector $x=\left(x_{n}\right)$ in $X$, we have $p_{q}(x)=$ $\left(x_{1}, \ldots, x_{q-1}, 0, x_{q+1}, \ldots\right)$. We can note that $\left\|p_{q}(x)\right\|=\max _{r \neq q}\left\|x_{r}\right\| \leq\|x\|$.

Let us define $L: X \rightarrow X_{n}$ by $L(0)=0$ and, if $x=\left(x_{n}\right)$ is different from 0 , then

$$
L(x)=\left(0, \ldots, 0,\left(1-\frac{\left\|p_{q}(x)\right\|}{\left\|x_{q}\right\|}\right) x_{q}, 0, \ldots\right)
$$

where $q$ is such that $\left\|x_{q}\right\|=\|x\|$.

This definition is correct since, if $\left\|x_{q}\right\|=\left\|x_{r}\right\|=\|x\|$ for $q \neq r$, then both calculations give $L(x)=0$.

If $x=\left(x_{n}\right)$ and $y=\left(y_{n}\right)$ are in $X$ and $\left\|x_{q}\right\|=\|x\|,\left\|y_{r}\right\|=\|y\|$ with $q \neq r$, then:

$$
\begin{aligned}
d(L(x), L(y)) & =\left(\left\|x_{q}\right\|-\left\|p_{q}(x)\right\|\right) \vee\left(\left\|y_{r}\right\|-\left\|p_{r}(y)\right\|\right) \\
& \leq\left(\left\|x_{q}\right\|-\left\|x_{r}\right\|\right) \vee\left(\left\|y_{r}\right\|-\left\|y_{q}\right\|\right) \\
& \leq\left\|x_{q}\right\|-\left\|y_{q}\right\|+\left\|y_{r}\right\|-\left\|x_{r}\right\| \leq 2\|x-y\| .
\end{aligned}
$$


Note that this includes the case $x=0$ or $y=0$. Now, if $\left\|x_{q}\right\|=\|x\|$ and $\left\|y_{r}\right\|=\|y\|$ for $q=r$, with $x \neq 0$ and $y \neq 0$, then

$$
\begin{aligned}
d(L(x), & L(y))=\left\|\left(1-\frac{\left\|p_{q}(x)\right\|}{\|x\|}\right) x_{q}-\left(1-\frac{\left\|p_{q}(y)\right\|}{\|y\|}\right) y_{q}\right\| \\
& \leq\left\|\left(1-\frac{\left\|p_{q}(x)\right\|}{\|x\|}\right)\left(x_{q}-y_{q}\right)\right\|+\left\|\left(\frac{\left\|p_{q}(y)\right\|}{\|y\|}-\frac{\left\|p_{q}(x)\right\|}{\|x\|}\right) y_{q}\right\| \\
& \leq\left\|x_{q}-y_{q}\right\|+\|y\|\left(\left|\frac{\left\|p_{q}(y)\right\|-\left\|p_{q}(x)\right\|}{\|y\|}\right|+\left|\frac{\left\|p_{q}(x)\right\|}{\|y\|}-\frac{\left\|p_{q}(x)\right\|}{\|x\|}\right|\right) \\
& \leq\|x-y\|+\left\|p_{q}(y-x)\right\|+\|y\|\left\|p_{q}(x)\right\|\left|\frac{1}{\|y\|}-\frac{1}{\|x\|}\right| \\
& \leq 2\|x-y\|+\frac{\left\|p_{q}(x)\right\|}{\|x\|}|\|x\|-\|y\|| \leq 3\|x-y\| .
\end{aligned}
$$

Hence $L$ is 3 -Lipschitz. Since $x \in \hat{X}_{n}$ implies $p_{n}(x)=0$, it is clear that $L$ is a retraction.

Lemma 3. Let $M$ be a metric space with a special point 0 and let $N$ be a subset of $M$ containing 0 . We suppose that there exists a Lipschitz-retraction $L$ from $M$ onto $N$ such that $L(0)=0$. Then $\mathcal{F}(N)$ is isomorphic to some complemented subspace of $\mathcal{F}(M)$.

Proof. Using Theorem 2.2.4.b in 10] (where $\mathcal{F}(M)$ is denoted by $\operatorname{AE}(X)$ ), we see that there exist linear maps $i: \mathcal{F}(N) \rightarrow \mathcal{F}(M)$ and $l: \mathcal{F}(M) \rightarrow \mathcal{F}(N)$ such that for any $x \in N, y \in M, f \in \operatorname{Lip}_{0}(M)$ and $g \in \operatorname{Lip}_{0}(N)$, we have $i\left(\delta_{x}\right)(f)=f(x)$ and $l\left(\delta_{y}\right)(g)=g(L(y))$. One can easily see that $i$ is a linear isomorphism from $\mathcal{F}(N)$ into $\mathcal{F}(M)$ and that $i l$ is a linear continuous projection from $\mathcal{F}(M)$ onto $i(\mathcal{F}(N))$.

Proposition 4. The spaces $\left(\bigoplus \mathcal{F}\left(c_{0}\right)\right)_{\ell_{1}}$ and $\mathcal{F}\left(c_{0}\right)$ are isomorphic.

Proof. Let $X_{1}, X_{2}, \ldots$ be Banach spaces. Then it follows from Lemmas 2 and 3 and Proposition 1 that the space $\left(\bigoplus \mathcal{F}\left(X_{n}\right)\right)_{\ell_{1}}$ is isomorphic to a complemented subspace of $\mathcal{F}\left(\left(\bigoplus X_{n}\right)_{c_{0}}\right)$. Assume now $X_{n}=c_{0}$ for each $n$. We infer that the spaces $\left(\bigoplus \mathcal{F}\left(c_{0}\right)\right)_{\ell_{1}}$ and $\mathcal{F}\left(c_{0}\right)$ are isomorphic to complemented subspaces of each other. Since $\left(\bigoplus \mathcal{F}\left(c_{0}\right)\right)_{\ell_{1}}$ is clearly isomorphic to its $\ell_{1}$ sum, the standard Pełczyński decomposition method shows that they are isomorphic.

Theorem 5. Let $K$ be any infinite metric compact space. The spaces $\mathcal{F}(C(K))$ and $\mathcal{F}\left(c_{0}\right)$ are isomorphic. Moreover the Banach-Mazur distance is uniformly bounded.

Proof. The space $c_{0}$ is 2-complemented in $C(K)$, so Lemma 3 shows that $\mathcal{F}\left(c_{0}\right)$ is isometric to a 2-complemented subspace of $\mathcal{F}(C(K))$.

By a classical result of Aharoni (see Theorem 7.11, p. 176 in [1]), we know that there is a 3-Lipschitz-homeomorphism between $C(K)$ and some subset of $c_{0}$. Since the spaces $C(K)$ are absolute Lipschitz retracts (with uniform constant), there is a Lipschitz retraction from $c_{0}$ onto $C(K)$ which maps 0 to 0 (with uniform constant). Using Lemma 3, we see that $\mathcal{F}(C(K))$ is isomorphic to a complemented subspace of $\mathcal{F}\left(c_{0}\right)$ (with uniform constants). Finally, Proposition 4 ensures that $\left(\bigoplus \mathcal{F}\left(c_{0}\right)\right)_{\ell_{1}}$ 
is isomorphic to $\mathcal{F}\left(c_{0}\right)$. Using the standard Pełczyński decomposition method, we infer that $\mathcal{F}\left(c_{0}\right)$ and $\mathcal{F}(C(K))$ are isomorphic with uniform constants.

Note that by the result of Aharoni, the space $\mathcal{F}\left(c_{0}\right)$ is universal for separable Banach spaces. However it is not isomorphic to $C(0,1)$ which is $\mathcal{L}^{\infty}$ whereas $L_{1}(0,1)$ is complemented in $\mathcal{F}\left(c_{0}\right)$.

An open question is whether a converse to Aharoni's result is true, that is, if $\mathcal{F}(X)$ is universal (equivalently contains $\mathcal{F}\left(c_{0}\right)$ ), does it follow that $c_{0}$ embeds into $X ?$ It is not even known if $X$ must contain the $\ell_{\infty}^{n}$ 's uniformly when $\mathcal{F}(X)$ is universal.

\section{LipsChitZ hOMOGENEOUS BANACH SPACES}

We include in this note a consequence of the results of Godefroy and Kalton on Lipschitz free Banach spaces. Define a Banach space to be Lipschitz homogeneous if it is Lipschitz homeomorphic to all its closed infinite-dimensional subspaces. The following question was asked in [2].

Question 6. Is every Lipschitz homogeneous Banach space isomorphic to $\ell_{2}$ ?

That the answer should be yes is a natural conjecture which would generalize the positive answer given to the homogeneous Banach space problem by Gowers 4 and Komorowski, Tomczak-Jaegermann [8. They proved that a Banach space which is isomorphic to its (closed infinite-dimensional) subspaces must be isomorphic to $\ell_{2}$. It is also related to the conjecture that for separable Banach spaces, Lipschitz homeomorphism and isomorphism coincide; if the latter is true, then obviously $\ell_{2}$ is the only Lipschitz homogeneous Banach space.

Using the results of Gowers and Komorowski, Tomczak-Jaegermann, partial positive answers to Question 6] were given in [2]: for example, if a dual Banach space is Lipschitz homogeneous, then it must be isomorphic to $\ell_{2}$.

We derive additional results about this problem using a result of Godefroy and Kalton [5] which states that the Bounded Approximation Property is stable under Lipschitz homeomorphism. We recall a few results about local unconditional structure (or l.u.st.). There are two notions of l.u.st. in the literature. The first, that we shall call l.u.st., was introduced by Figiel, Johnson and Tzafriri [3], on the model of $L_{p}$-spaces. The second was introduced by Gordon and Lewis [6], and we shall write it GL-l.u.st. For the definition:

Definition 7. A Banach space $X$ is said to have l.u.st. if there is a constant $C \geq 1$ and an increasing net $E_{\alpha}$ of finite-dimensional subspaces of $X$, such that $X=\overline{\bigcup_{\alpha} E_{\alpha}}$ and such that for every $\alpha, E_{\alpha}$ has a $C$-unconditional basis.

A Banach space $X$ is said to have GL-l.u.st. if there is a constant $C \geq 1$ such that for every finite-dimensional subspace $E$ of $X$, there exists a finite-dimensional space $F$ with a 1-unconditional basis, and maps $T: E \rightarrow F, U: F \rightarrow X$, such that $U T(x)=x$ for all $x \in E$ and such that $\|T\|\|U\| \leq C$.

While it is clear that l.u.st. implies GL-l.u.st., the converse is only known to hold in some special cases. The following proposition is proved in [3] with l.u.st. instead of GL-l.u.st. But we notice that gathering some facts in that article allows us to generalize it to Banach spaces with GL-l.u.st. 
Proposition 8. Let $X$ be a Banach space with GL-l.u.st. which does not contain $\ell_{\infty}^{n}$ 's uniformly. Then every subspace of $X$ has a further subspace with an unconditional basis.

Proof. Assume $X$ has GL-l.u.st. Then by [3, Remark 2.3, $X^{* *}$ is complemented in a Banach lattice $L$. So $X^{(4)}$ (and thus $X^{* *}$ ) is complemented in $L^{* *}$ which is a $\sigma$ complete Banach lattice. By local reflexivity, $X^{* *}$ does not contain $\ell_{\infty}^{n}$ 's uniformly, so by $\left[3\right.$, Proposition 2.6, $X^{* *}$ is complemented in a Banach lattice $M$ which does not contain $\ell_{\infty}^{n}$ 's uniformly. In particular $M$ does not contain a copy of $c_{0}$, so is $\sigma$-complete and $\sigma$-order continuous, and $X$ is isomorphic to a subspace of $M$. Thus we may apply [3], Theorem 4.1, to conclude that every subspace of $X$ contains an unconditional basic sequence.

The following proposition mixes new results with some results previously obtained in [2].

Proposition 9. Let $X$ be a Lipschitz homogeneous Banach space which is not isomorphic to $\ell_{2}$. Then

(a) no subspace of $X$ has the Radon-Nikodým Property,

(b) no subspace of $X$ has GL-l.u.st.,

(c) $X$ has a hereditarily indecomposable subspace,

(d) every subspace of $X$ has the Bounded Approximation Property,

(e) $X$ has cotype $2+\epsilon$ and type $2-\epsilon$ for any $\epsilon>0$.

Proof. (a) was proved in [2], where it was also proved that $X$ does not contain a subspace with an unconditional basis, and so by Gowers' dichotomy theorem, (c) follows. Every Banach space has a subspace with a Schauder basis, and so (d) follows from the result of Godefroy and Kalton that we recalled. (e) follows from (d) and the results of Szankowski about the Approximation Property (see e.g. 9]). By (e), $X$ does not contain $\ell_{\infty}^{n}$ 's uniformly and so (b) follows from the previous proposition.

Properties (a)-(e) are a strange combination of positive and negative results, and of course, no example of a Banach space satisfying (a)-(e) is known. The task of finding such a space with the additional property of being Lipschitz homogeneous seems to be out of reach. This suggests that $\ell_{2}$ should be the only Lipschitz homogeneous Banach space.

\section{REFERENCES}

[1] Y. Benyamini and J. Lindenstrauss, Geometric nonlinear functional analysis. Vol. 1, Amer. Math. Soc., Providence, RI, 2000. MR1727673 (2001b:46001)

[2] V. Ferenczi, Lipschitz homogeneous Banach spaces, Q. J. Math. 54 (2003), no. 4, 415-419. MR2031174

[3] T. Figiel, W. B. Johnson and L. Tzafriri, On Banach lattices and spaces having local unconditional structure, with applications to Lorentz function spaces, J. Approximation Theory 13 (1975), 395-412. MR0367624(51:3866)

[4] W. T. Gowers, An infinite Ramsey theorem and some Banach-space dichotomies, Ann. of Math. (2) 156 (2002), no. 3, 797-833. MR.1954235

[5] G. Godefroy and N. J. Kalton, Lipschitz-free Banach spaces, Studia Math. 159 (2003), no. 1, 121-141. MR2030906 (2004m:46027)

[6] Y. Gordon and D. R. Lewis, Absolutely summing operators and local unconditional structures, Acta Math. 133 (1974), 27-48. MR0410341(53:14091) 
[7] W. B. Johnson, J. Lindenstrauss and G. Schechtman, Banach spaces determined by their uniform structures, Geom. Funct. Anal. 6 (1996), no. 3, 430-470. MR1392325 (97b:46016)

[8] R. A. Komorowski and N. Tomczak-Jaegermann, Banach spaces without local unconditional structure, Israel J. Math. 89 (1995), no. 1-3, 205-226. MR1324462 (96g:46007) Erratum to: "Banach spaces without local unconditional structure", Israel J. Math. 105 (1998), 85-92. MR:1639731 (99g:46008)

[9] J. Lindenstrauss and L. Tzafriri, Classical Banach spaces. I, Springer, Berlin, 1977. MR0500056 (58:17766)

[10] N. Weaver, Lipschitz algebras, World Scientific Publishing Co., Inc., River Edge, NJ, 1999. MR.1832645 (2002g:46002)

Université de Franche-Comté, Laboratoire de Mathématiques, 16 route de Gray, 25030 Besançon Cedex, France

E-mail address: dutrieux@math.univ-fcomte.fr

Institut de Mathématiques, Analyse Fonctionnelle, Université Paris 6, Boîte 186, 4 Place Jussieu, 75252 Paris Cedex 05, France

E-mail address: ferenczi@ccr.jussieu.fr 